\title{
Positive Solutions for a System of Fractional Differential Equations with Parameters and Coupled Multi-point Boundary Conditions
}

\author{
Johnny Henderson ${ }^{1}$, Rodica Luca ${ }^{2}$, Alexandru Tudorache ${ }^{3}$ \\ ${ }^{1}$ Baylor University, Department of Mathematics, Waco, Texas, 76798-7328 USA. \\ ${ }^{2}$ Gh. Asachi Technical University, Department of Mathematics, Iasi 700506, Romania. \\ ${ }^{3}$ Gh. Asachi Technical University, Faculty of Computer Engineering and Automatic Control, Iasi 700050, \\ Romania.
}

* Corresponding author. Tel.: 0040749188962; email: rluca@math.tuiasi.ro rlucatudor@yahoo.com Manuscript submitted May 6, 2018; accepted July 9, 2018.

doi: 10.17706/ijapm.2018.8.4.53-65

\begin{abstract}
In this paper, we study a system of nonlinear Riemann-Liouville fractional ordinary differential equations with parameters, subject to coupled multi-point boundary conditions which contain fractional derivatives. By using some properties of the associated Green's functions and the Guo-Krasnosel'skii fixed point theorem, we prove the existence of positive solutions for this problem when the parameters belong to various intervals. Then, we present sufficient conditions for the nonexistence of positive solutions.
\end{abstract}

Key words: Fractional differential equations, multi-point boundary conditions, positive solutions, existence, nonexistence.

\section{Introduction}

We consider the system of nonlinear ordinary fractional differential equations

$$
\begin{cases}D_{0+}^{\alpha} u(t)+\lambda f(t, u(t), v(t))=0, & t \in(0,1) \\ D_{0+}^{\beta} v(t)+\mu g(t, u(t), v(t))=0, & t \in(0,1)\end{cases}
$$

with the coupled multi-point boundary conditions

$$
\left\{\begin{array}{l}
u^{(j)}(0)=0, j=0, \ldots, n-2 ;\left.D_{0+}^{p_{1}} u(t)\right|_{t=1}=\left.\sum_{i=1}^{N} a_{i} D_{0+}^{q_{1}} v(t)\right|_{t=\xi_{i}} \\
v^{(j)}(0)=0, j=0, \ldots, m-2 ;\left.\quad D_{0+}^{p_{2}} v(t)\right|_{t=1}=\left.\sum_{i=1}^{M} b_{i} D_{0+}^{q_{2}} u(t)\right|_{t=\eta_{i}}
\end{array}\right.
$$

where $\quad \alpha, \beta \in \mathbb{R}, \alpha \in(n-1, n], \beta \in(m-1, m], n, m \in \mathbb{N}, n, m \geq 3, p_{1}, p_{2}, q_{1}, q_{2} \in \mathbb{R}, p_{1} \in[1, n-2], p_{2} \in$ $[1, m-2], q_{1} \in\left[0, p_{2}\right], q_{2} \in\left[0, p_{1}\right], \xi_{i}, a_{i} \in \mathbb{R}$ for all $i=1, \ldots, N(N \in \mathbb{N}), 0<\xi_{1}<\cdots<\xi_{N} \leq 1, \eta_{i}, b_{i} \in \mathbb{R}$ for all $i=1, \ldots, M(M \in \mathbb{N}), 0<\eta_{1}<\cdots<\eta_{M} \leq 1, \lambda, \mu>0$, and $D_{0+}^{k}$ denotes the Riemann-Liouville derivative of order $k$ (for $k=\alpha, \beta, p_{1}, p_{2}, q_{1}, q_{2}$ ).

Under some assumptions on the nonnegative functions $f$ and $g$, we present intervals for the parameters $\lambda$ and $\mu$ such that positive solutions of $(S)-(B C)$ exist. By a positive solution of problem (S)-(BC) we mean a pair of functions $(u, v) \in(C([0,1]),[0, \infty)))^{2}$ satisfying $(S)$ and $(B C)$, with $u(t)>0$ for all 
$t \in(0,1]$ or $v(t)>0$ for all $t \in(0,1]$. The nonexistence of positive solutions for (S)-(BC) is also investigated. The system $(S)$ without parameters $\lambda$, $\mu$ with the boundary conditions $(B C)$ was investigated in [1], where $f$ and $g$ are non-singular or singular functions. The existence of positive solutions of the system (S) without parameters subject to the uncoupled multi-point boundary conditions

$$
\left\{\begin{array}{l}
u^{(j)}(0)=0, j=0, \ldots, n-2 ;\left.\quad D_{0+}^{p_{1}} u(t)\right|_{t=1}=\left.\sum_{i=1}^{N} a_{i} D_{0+}^{q_{1}} u(t)\right|_{t=\xi_{i}}, \\
v^{(j)}(0)=0, j=0, \ldots, m-2 ;\left.D_{0+}^{p_{2}} v(t)\right|_{t=1}=\left.\sum_{i=1}^{M} b_{i} D_{0+}^{q_{2}} v(t)\right|_{t=\eta_{i}},
\end{array}\right.
$$

was studied in [2], [3]. For other papers which investigate the existence, nonexistence and multiplicity of positive solutions for systems of fractional differential equations with nonnegative or sign-changing nonlinearities, subject to various nonlocal boundary conditions we mention [4]-[12].

Fractional differential equations describe many phenomena in several fields of engineering and scientific disciplines such as physics, biophysics, chemistry, biology (for example, the primary infection with HIV), economics, control theory, signal and image processing, thermoelasticity, aerodynamics, viscoelasticity, electromagnetics and rheology (see [13-28]). Fractional differential equations are also regarded as a better tool for the description of hereditary properties of various materials and processes than the corresponding integer order differential equations.

The paper is organized as follows. In Section 2, we present some auxiliary results which investigate a nonlocal boundary value problem for fractional differential equations, and we give the properties of the Green functions associated to our problem. Section 3 contains the existence and nonexistence results for the positive solutions of problem (S)-(BC). In the proof of our main existence theorems we use the Guo-Krasnosel'skii fixed point theorem (see [29]). Finally, in Section 4, an example is given to support the new results.

\section{Auxiliary Results}

In this section we present some auxiliary results from [1] that will be used to prove our main theorems.

We consider the fractional differential system

$$
\left\{\begin{array}{l}
D_{0+}^{\alpha} u(t)+x(t)=0, \quad t \in(0,1) \\
D_{0+}^{\beta} v(t)+y(t)=0, \quad t \in(0,1)
\end{array}\right.
$$

with the coupled multi-point boundary conditions (BC), where $\alpha, \beta \in \mathbb{R}, \alpha \in(n-1, n], \beta \in(m-1, m]$, $n, m \in \mathbb{N}, n, m \geq 3, p_{1}, p_{2}, q_{1}, q_{2} \in \mathbb{R}, p_{1} \in[1, n-2], p_{2} \in[1, m-2], q_{1} \in\left[0, p_{2}\right], q_{2} \in\left[0, p_{1}\right], \xi_{i}, a_{i} \in \mathbb{R}$ for all $i=1, \ldots, N(N \in \mathbb{N}), \quad 0<\xi_{1}<\cdots<\xi_{N} \leq 1, \eta_{i}, b_{i} \in \mathbb{R}$ for all $i=1, \ldots, M(M \in \mathbb{N}), 0<\eta_{1}<\cdots<\eta_{M} \leq$ 1 , and $x, y:(0,1) \rightarrow \mathbb{R}$ are continuous functions.

We denote by $\Delta$ the constant

$$
\Delta=\frac{\Gamma(\alpha) \Gamma(\beta)}{\Gamma\left(\alpha-p_{1}\right) \Gamma\left(\beta-p_{2}\right)}-\frac{\Gamma(\alpha) \Gamma(\beta)}{\Gamma\left(\alpha-q_{2}\right) \Gamma\left(\beta-q_{1}\right)}\left(\sum_{i=1}^{N} a_{i} \xi_{i}^{\beta-q_{1}-1}\right)\left(\sum_{i=1}^{M} b_{i} \eta_{i}^{\alpha-q_{2}-1}\right)
$$

Lemma 2.1 If $\Delta \neq 0$ and $x, y \in C(0,1) \cap L^{1}(0,1)$, then the pair of functions $(u, v) \in C[0,1] \times C[0,1]$ given by 


$$
\begin{cases}u(t)=\int_{0}^{1} G_{1}(t, s) x(s) d s+\int_{0}^{1} G_{2}(t, s) y(s) d s, & t \in[0,1] \\ v(t)=\int_{0}^{1} G_{3}(t, s) y(s) d s+\int_{0}^{1} G_{4}(t, s) x(s) d s, & t \in[0,1]\end{cases}
$$

is solution of problem (1)-(BC), where

$$
\begin{gathered}
G_{1}(t, s)=g_{1}(t, s)+\frac{t^{\alpha-1} \Gamma(\beta)}{\Delta \Gamma\left(\beta-q_{1}\right)}\left(\sum_{i=1}^{N} a_{i} \xi_{i}^{\beta-q_{1}-1}\right)\left(\sum_{i=1}^{M} b_{i} g_{2}\left(\eta_{i}, s\right)\right), \\
G_{2}(t, s)=\frac{t^{\alpha-1} \Gamma(\beta)}{\Delta \Gamma\left(\beta-p_{2}\right)}\left(\sum_{i=1}^{N} a_{i} g_{3}\left(\xi_{i}, s\right)\right) \\
G_{3}(t, s)=g_{4}(t, s)+\frac{t^{\beta-1} \Gamma(\alpha)}{\Delta \Gamma\left(\alpha-q_{2}\right)}\left(\sum_{i=1}^{M} b_{i} \eta_{i}^{\alpha-q_{2}-1}\right)\left(\sum_{i=1}^{N} a_{i} g_{3}\left(\xi_{i}, s\right)\right) \\
G_{4}(t, s)=\frac{t^{\beta-1} \Gamma(\alpha)}{\Delta \Gamma\left(\alpha-p_{1}\right)}\left(\sum_{i=1}^{M} b_{i} g_{2}\left(\eta_{i}, s\right)\right), \quad \forall t, s \in[0,1]
\end{gathered}
$$

And

$$
\begin{gathered}
g_{1}(t, s)=\frac{1}{\Gamma(\alpha)}\left\{\begin{array}{c}
t^{\alpha-1}(1-s)^{\alpha-p_{1}-1}-(t-s)^{\alpha-1}, 0 \leq s \leq t \leq 1, \\
t^{\alpha-1}(1-s)^{\alpha-p_{1}-1}, 0 \leq t \leq s \leq 1,
\end{array}\right. \\
g_{2}(t, s)=\frac{1}{\Gamma\left(\alpha-q_{2}\right)}\left\{\begin{array}{r}
t^{\alpha-q_{2}-1}(1-s)^{\alpha-p_{1}-1}-(t-s)^{\alpha-q_{2}-1}, 0 \leq s \leq t \leq 1, \\
t^{\alpha-q_{2}-1}(1-s)^{\alpha-p_{1}-1}, 0 \leq t \leq s \leq 1 .
\end{array}\right. \\
g_{3}(t, s)=\frac{1}{\Gamma\left(\beta-q_{1}\right)}\left\{\begin{array}{c}
t^{\beta-q_{1}-1}(1-s)^{\beta-p_{2}-1}-(t-s)^{\beta-q_{1}-1}, 0 \leq s \leq t \leq 1, \\
t^{\beta-q_{1}-1}(1-s)^{\beta-p_{2}-1}, 0 \leq t \leq s \leq 1 .
\end{array}\right. \\
g_{4}(t, s)=\frac{1}{\Gamma(\beta)}\left\{\begin{array}{c}
t^{\beta-1}(1-s)^{\beta-p_{2}-1}-(t-s)^{\beta-1}, 0 \leq s \leq t \leq 1, \\
t^{\beta-1}(1-s)^{\beta-p_{2}-1}, 0 \leq t \leq s \leq 1 .
\end{array}\right.
\end{gathered}
$$

Lemma 2.2 The functions $g_{i}, 1=1, \ldots, 4$ given by (5) have the properties:

$\left.a_{1}\right) g_{1}(t, s) \leq h_{1}(s)$ for all $t, s \in[0,1]$, where $h_{1}(s)=\frac{1}{\Gamma(\alpha)}(1-s)^{\alpha-p_{1}-1}\left(1-(1-s)^{p_{1}}\right), s \in[0,1]$;

$\left.a_{2}\right) g_{1}(t, s) \geq t^{\alpha-1} h_{1}(s)$ for all $t, s \in[0,1]$;

$\left.a_{3}\right) g_{1}(t, s) \leq \frac{1}{\Gamma(\alpha)} t^{\alpha-1}$ for all $t, s \in[0,1]$

$\left.b_{1}\right) g_{2}(t, s) \geq t^{\alpha-q_{2}-1} h_{2}(s)$ for all $t, s \in[0,1]$, where

$$
h_{2}(s)=\frac{1}{\Gamma\left(\alpha-q_{2}\right)}(1-s)^{\alpha-p_{1}-1}\left(1-(1-s)^{p_{1}-q_{2}}\right), s \in[0,1]
$$

$\left.b_{2}\right) g_{2}(t, s) \leq \frac{1}{\Gamma\left(\alpha-q_{2}\right)} t^{\alpha-q_{2}-1}(1-s)^{\alpha-p_{1}-1}$ for all $t, s \in[0,1]$;

$\left.b_{3}\right) g_{2}(t, s) \leq \frac{1}{\Gamma\left(\alpha-q_{2}\right)} t^{\alpha-q_{2}-1}$ for all $t, s \in[0,1]$;

$\left.c_{1}\right) g_{1}(t, s) \geq t^{\beta-q_{1}-1} h_{3}(s)$ for all $t, s \in[0,1]$, where

$$
h_{3}(s)=\frac{1}{\Gamma\left(\beta-q_{1}\right)}(1-s)^{\beta-p_{2}-1}\left(1-(1-s)^{p_{2}-q_{1}}\right), s \in[0,1]
$$

$\left.c_{2}\right) g_{3}(t, s) \leq \frac{1}{\Gamma\left(\beta-q_{1}\right)} t^{\beta-q_{1}-1}(1-s)^{\beta-p_{2}-1}$ for all $t, s \in[0,1]$;

$\left.c_{3}\right) g_{3}(t, s) \leq \frac{1}{\Gamma\left(\beta-q_{1}\right)} t^{\beta-q_{1}-1}$ for all $t, s \in[0,1]$;

$\left.d_{1}\right) g_{4}(t, s) \leq h_{4}(s)$ for all $t, s \in[0,1]$, where $h_{4}(s)=\frac{1}{\Gamma(\beta)}(1-s)^{\beta-p_{2}-1}\left(1-(1-s)^{p_{2}}\right), s \in[0,1]$;

$\left.d_{2}\right) g_{4}(t, s) \geq t^{\beta-1} h_{4}(s)$ for all $t, s \in[0,1]$

$\left.d_{3}\right) g_{4}(t, s) \leq \frac{1}{\Gamma(\beta)} t^{\beta-1}$ for all $t, s \in[0,1]$; 
e) The functions $g_{i}, 1=1, \ldots, 4$ are continuous on $[0,1] \times[0,1] ; g_{i}(t, s) \geq 0$, for all $t, s \in[0,1]$; $g_{i}(t, s)>0$, for all $t, s \in(0,1), i=1, \ldots, 4$.

Lemma 2.3 If $\Delta>0, a_{i} \geq 0$ for all $i=1, \ldots, N$, and $b_{i} \geq 0$ for all $i=1, \ldots, M$, then the functions $G_{i}, i=1, \ldots, 4$ given by (4) have the properties

$\left.a_{1}\right) G_{1}(t, s) \leq J_{1}(s), \forall(t, s) \in[0,1] \times[0,1]$, where

$$
J_{1}(s)=h_{1}(s)+\frac{\Gamma(\beta)}{\Delta \Gamma\left(\beta-q_{1}\right)}\left(\sum_{i=1}^{N} a_{i} \xi_{i}^{\beta-q_{1}-1}\right)\left(\sum_{i=1}^{M} b_{i} g_{2}\left(\eta_{i}, s\right)\right), \forall s \in[0,1] ;
$$

$\left.a_{2}\right) G_{1}(t, s) \geq t^{\alpha-1} J_{1}(s), \forall(t, s) \in[0,1] \times[0,1]$;

$\left.a_{3}\right) G_{1}(t, s) \leq \delta_{1} t^{\alpha-1}, \forall(t, s) \in[0,1] \times[0,1]$, where

$$
\delta_{1}=\frac{1}{\Gamma(\alpha)}+\frac{\Gamma(\beta)}{\Delta \Gamma\left(\beta-q_{1}\right) \Gamma\left(\alpha-q_{2}\right)}\left(\sum_{i=1}^{N} a_{i} \xi_{i}^{\beta-q_{1}-1}\right)\left(\sum_{i=1}^{M} b_{i} \eta_{i}^{\alpha-q_{2}-1}\right) ;
$$

$\left.b_{1}\right) G_{2}(t, s) \leq J_{2}(s), \forall(t, s) \in[0,1] \times[0,1]$, where $J_{2}(s)=\frac{\Gamma(\beta)}{\Delta \Gamma\left(\beta-p_{2}\right)}\left(\sum_{i=1}^{N} a_{i} g_{3}\left(\xi_{i}, s\right)\right), \forall s \in[0,1]$;

$\left.b_{2}\right) G_{2}(t, s)=t^{\alpha-1} J_{2}(s), \forall(t, s) \in[0,1] \times[0,1]$;

$\left.b_{3}\right) G_{2}(t, s) \leq \delta_{2} t^{\alpha-1}, \forall(t, s) \in[0,1] \times[0,1]$, where $\delta_{2}=\frac{\Gamma(\beta)}{\Delta \Gamma\left(\beta-p_{2}\right) \Gamma\left(\beta-q_{1}\right)} \sum_{i=1}^{N} a_{i} \xi_{i}^{\beta-q_{1}-1}$;

$\left.c_{1}\right) G_{3}(t, s) \leq J_{3}(s), \forall(t, s) \in[0,1] \times[0,1]$, where

$$
J_{3}(s)=h_{4}(s)+\frac{\Gamma(\alpha)}{\Delta \Gamma\left(\alpha-q_{2}\right)}\left(\sum_{i=1}^{M} b_{i} \eta_{i}^{\alpha-q_{2}-1}\right)\left(\sum_{i=1}^{N} a_{i} g_{3}\left(\xi_{i}, s\right)\right), \forall s \in[0,1] ;
$$

$\left.c_{2}\right) G_{3}(t, s) \geq t^{\beta-1} J_{3}(s), \forall(t, s) \in[0,1] \times[0,1]$;

$\left.c_{3}\right) G_{3}(t, s) \leq \delta_{3} t^{\beta-1}, \forall(t, s) \in[0,1] \times[0,1]$, where

$$
\delta_{3}=\frac{1}{\Gamma(\beta)}+\frac{\Gamma(\alpha)}{\Delta \Gamma\left(\alpha-q_{2}\right) \Gamma\left(\beta-q_{1}\right)}\left(\sum_{i=1}^{M} b_{i} \eta_{i}^{\alpha-q_{2}-1}\right)\left(\sum_{i=1}^{N} a_{i} \xi_{i}^{\beta-q_{1}-1}\right) ;
$$

$\left.d_{1}\right) G_{4}(t, s) \leq J_{4}(s), \forall(t, s) \in[0,1] \times[0,1]$, where $J_{4}(s)=\frac{\Gamma(\alpha)}{\Delta \Gamma\left(\alpha-p_{1}\right)}\left(\sum_{i=1}^{M} b_{i} g_{2}\left(\eta_{i}, s\right)\right), \forall s \in[0,1]$;

$\left.d_{2}\right) G_{4}(t, s)=t^{\beta-1} J_{4}(s), \forall(t, s) \in[0,1] \times[0,1]$;

$\left.d_{3}\right) G_{4}(t, s) \leq \delta_{4} t^{\beta-1}, \forall(t, s) \in[0,1] \times[0,1]$, where $\delta_{4}=\frac{\Gamma(\alpha)}{\Delta \Gamma\left(\alpha-p_{1}\right) \Gamma\left(\alpha-q_{2}\right)} \sum_{i=1}^{M} b_{i} \eta_{i}^{\alpha-q_{2}-1}$.

e) The functions $G_{i}, 1=1, \ldots, 4$ are continuous on $[0,1] \times[0,1]$, and $G_{i}(t, s) \geq 0$, for all $t, s \in[0,1]$, $i=1, \ldots, 4$.

Lemma 2.4 If $\Delta>0, a_{i} \geq 0$ for all $i=1, \ldots, N, b_{i} \geq 0$ for all $i=1, \ldots, M$, and $x, y \in C(0,1) \cap L^{1}(0,1)$ with $x(t) \geq 0, y(t) \geq 0$ for all $t \in(0,1)$, then the solution $(u, v)$ of problem (1)-(BC) given by (3) satisfies the inequalities $u(t) \geq 0, v(t) \geq 0$ for all $t \in[0,1]$. Moreover, we have the inequalities $u(t) \geq$ $t^{\alpha-1} u\left(t^{\prime}\right)$ and $v(t) \geq t^{\beta-1} v\left(t^{\prime}\right)$ for all $t, t^{\prime} \in[0,1]$.

Remark 2.1 Under the assumptions of Lemma 2.4, for any interval $\left[c_{1}, c_{2}\right] \subset[0,1]$ with $0<c_{1}<c_{2} \leq 1$, the solution of problem (1)-(BC) given by (3) satisfies the inequalities $\min _{t \in\left[c_{1}, c_{2}\right]} u(t) \geq c_{1}^{\alpha-1} \max _{t^{\prime} \in[0,1]} u\left(t^{\prime}\right)$ and $\min _{t \in\left[c_{1}, c_{2}\right]} v(t) \geq c_{1}^{\beta-1} \max _{t^{\prime} \in[0,1]} v\left(t^{\prime}\right)$.

In the proof of our main existence results we will use the Guo-Krasnosel'skii fixed point theorem presented below (see [29]).

Theorem 2.1 Let X be a Banach space and let $C \subset X$ be a cone in X. Assume $\Omega_{1}$ and $\Omega_{2}$ are bounded open subsets of X with $0 \in \Omega_{1} \subset \overline{\Omega_{1}} \subset \Omega_{2}$ and let $\mathrm{A}: C \cap\left(\overline{\Omega_{2}} \backslash \Omega_{1}\right) \rightarrow C$ be a completely continuous operator such that, either

1) $\quad\|A u\| \leq\|u\|, u \in C \cap \partial \Omega_{1}$, and $\|A u\| \geq\|u\|, u \in C \cap \partial \Omega_{2}$, or

2) $\quad\|A u\| \geq\|u\|, u \in C \cap \partial \Omega_{1}$, and $\|A u\| \leq\|u\|, u \in C \cap \partial \Omega_{2}$.

Then $A$ has a fixed point in $C \cap\left(\overline{\Omega_{2}} \backslash \Omega_{1}\right)$.

\section{Main Results}

In this section we give first some sufficient conditions on $\lambda, \mu, f$ and $g$ such that positive solutions with respect to a cone for our problem $(S)-(B C)$ exist. 
We present now the assumptions that we will use in the sequel.

(H1) $\alpha, \beta \in \mathbb{R}, \alpha \in(n-1, n], \beta \in(m-1, m], n, m \in \mathbb{N}, n, m \geq 3, p_{1}, p_{2}, q_{1}, q_{2} \in \mathbb{R}, p_{1} \in[1, n-2], p_{2} \in$ $[1, m-2], q_{1} \in\left[0, p_{2}\right], q_{2} \in\left[0, p_{1}\right], \xi_{i} \in \mathbb{R}, a_{i} \geq 0$ for all $i=1, \ldots, N(N \in \mathbb{N}), 0<\xi_{1}<\cdots<\xi_{N} \leq 1, \eta_{i} \in$ $\mathbb{R}, b_{i} \geq 0$ for all $i=1, \ldots, M(M \in \mathbb{N}), 0<\eta_{1}<\cdots<\eta_{M} \leq 1, \lambda, \mu>0$ and $\Delta>0$ (given by (2)).

(H2) The functions $f, g:[0,1] \times[0, \infty) \times[0, \infty) \rightarrow[0, \infty)$ are continuous.

For $\left[c_{1}, c_{2}\right] \subset[0,1]$ with $0<c_{1}<c_{2} \leq 1$, we introduce the following extreme limits

$$
\begin{aligned}
& f_{0}^{s}=\limsup _{u+v \rightarrow 0^{+}} \max _{t \in[0,1]} \frac{f(t, u, v)}{u+v}, \quad g_{0}^{s}=\limsup _{u+v \rightarrow 0^{+}} \max _{t \in[0,1]} \frac{g(t, u, v)}{u+v}, \\
& f_{0}^{i}=\liminf _{u+v \rightarrow 0^{+}} \min _{t \in\left[c_{1}, c_{2}\right]} \frac{f(t, u, v)}{u+v}, \quad g_{0}^{i}=\liminf _{u+v \rightarrow 0^{+}} \min _{t \in\left[c_{1}, c_{2}\right]} \frac{g(t, u, v)}{u+v}, \\
& f_{\infty}^{s}=\limsup _{u+v \rightarrow \infty} \max _{t \in[0,1]} \frac{f(t, u, v)}{u+v}, \quad g_{\infty}^{s}=\limsup _{u+v \rightarrow \infty} \max _{t \in[0,1]} \frac{g(t, u, v)}{u+v}, \\
& f_{\infty}^{i}=\liminf _{u+v \rightarrow \infty} \min _{t \in\left[c_{1}, c_{2}\right]} \frac{f(t, u, v)}{u+v}, \quad g_{\infty}^{i}=\liminf _{u+v \rightarrow \infty} \min _{t \in\left[c_{1}, c_{2}\right]} \frac{g(t, u, v)}{u+v} .
\end{aligned}
$$

In the definitions of the extreme limits above the variables $u$ and $v$ are nonnegative.

By using Lemma 2.1, a solution of the following nonlinear system of integral equations

$$
\begin{cases}u(t)=\lambda \int_{0}^{1} G_{1}(t, s) f(s, u(s), v(s)) d s+\mu \int_{0}^{1} G_{2}(t, s) g(s, u(s), v(s)) d s, & t \in[0,1] \\ v(t)=\mu \int_{0}^{1} G_{3}(t, s) g(s, u(s), v(s)) d s+\lambda \int_{0}^{1} G_{4}(t, s) f(s, u(s), v(s)) d s, & t \in[0,1]\end{cases}
$$

is solution of problem $(S)-(B C)$.

We consider the Banach space $X=C[0,1]$ with the supremum norm $\|\cdot\|$, and the Banach space $Y=X \times X$ with the norm $\|(u, v)\|_{Y}=\|u\|+\|v\|$. We define the cone $P \subset Y$ by

$$
P=\left\{(u, v) \in Y ; u(t) \geq t^{\alpha-1}\|u\|, \quad v(t) \geq t^{\beta-1}\|v\|, \quad \forall t \in[0,1]\right\} .
$$

For $\lambda, \mu>0$, we introduce the operators $Q_{1}, Q_{2}: Y \rightarrow X$ and $Q: Y \rightarrow Y$ defined by

$$
\begin{aligned}
& Q_{1}(u, v)(t)=\lambda \int_{0}^{1} G_{1}(t, s) f(s, u(s), v(s)) d s+\mu \int_{0}^{1} G_{2}(t, s) g(s, u(s), v(s)) d s, \quad t \in[0,1], \\
& Q_{2}(u, v)(t)=\mu \int_{0}^{1} G_{3}(t, s) g(s, u(s), v(s)) d s+\lambda \int_{0}^{1} G_{4}(t, s) f(s, u(s), v(s)) d s, \quad t \in[0,1],
\end{aligned}
$$

and $Q(u, v)=\left(Q_{1}(u, v), Q_{2}(u, v)\right),(u, v) \in Y$. Then if $(u, v)$ is a fixed point of operator $Q$, then $(u, v)$ is a solution of problem $(S)-(B C)$. Using Lemma 2.4 and similar arguments as those used in the proof of Lemma 3.1 from [5], we deduce that under assumptions (H1)-(H2), the operator $Q: P \rightarrow P$ is a completely continuous operator.

For $\quad\left[c_{1}, c_{2}\right] \subset[0,1] \quad$ with $0<c_{1}<c_{2} \leq 1 \quad$ we denote by 
$A=\int_{0}^{1} J_{1}(s) d s, B=\int_{0}^{1} J_{2}(s) d s, C=\int_{0}^{1} J_{3}(s) d s, D=\int_{0}^{1} J_{4}(s) d s, \tilde{A}=\int_{c_{1}}^{c_{2}} J_{1}(s) d s, \tilde{B}=\int_{c_{1}}^{c_{2}} J_{2}(s) d s, \tilde{C}=$ $\int_{c_{1}}^{c_{2}} J_{3}(s) d s, \widetilde{D}=\int_{c_{1}}^{c_{2}} J_{4}(s) d s$, where $J_{i}, i=1, \ldots, 4$ are defined in Section 2 (Lemma 2.3).

For $f_{0}^{s}, g_{0}^{s}, f_{\infty}^{i}, g_{\infty}^{i} \in(0, \infty)$ and numbers $\alpha_{1}, \alpha_{2} \in[0,1], \alpha_{3}, \alpha_{4} \in(0,1), a \in[0,1]$ and $b \in(0,1)$, we define the numbers

$$
\begin{gathered}
L_{1}=\max \left\{\frac{a \alpha_{1}}{\gamma \gamma_{1} f_{\infty}^{i} \tilde{A}}, \frac{(1-a) \alpha_{2}}{\gamma \gamma_{2} f_{\infty}^{i} \widetilde{D}}\right\}, \quad L_{2}=\min \left\{\frac{b \alpha_{3}}{f_{0}^{s} A}, \frac{(1-b) \alpha_{4}}{f_{0}^{s} D}\right\}, \\
L_{3}=\max \left\{\frac{a\left(1-\alpha_{1}\right)}{\gamma \gamma_{1} g_{\infty}^{i} \tilde{B}}, \frac{(1-a)\left(1-\alpha_{2}\right)}{\gamma \gamma_{2} g_{\infty}^{i} \tilde{C}}\right\}, \quad L_{4}=\min \left\{\frac{b\left(1-\alpha_{3}\right)}{g_{0}^{S} B}, \frac{(1-b)\left(1-\alpha_{4}\right)}{g_{0}^{s} C}\right\}, \\
L_{2}^{\prime}=\min \left\{\frac{b}{f_{0}^{s} A}, \frac{1-b}{f_{0}^{s} D}\right\}, \quad L_{4}^{\prime}=\min \left\{\frac{b}{g_{0}^{s} B}, \frac{1-b}{g_{0}^{s} C}\right\},
\end{gathered}
$$

where $\gamma_{1}=c_{1}^{\alpha-1}, \gamma_{2}=c_{1}^{\beta-1}$ and $\gamma=\min \left\{\gamma_{1}, \gamma_{2}\right\}$.

Theorem 3.1 Assume that (H1) and (H2) hold, $\left[c_{1}, c_{2}\right] \subset[0,1]$ with $0<c_{1}<c_{2} \leq 1, \alpha_{1}, \alpha_{2} \in[0,1]$, $\alpha_{3}, \alpha_{4} \in(0,1), a \in[0,1]$ and $b \in(0,1)$.

1) If $f_{0}^{s}, g_{0}^{s}, f_{\infty}^{i}, g_{\infty}^{i} \in(0, \infty), L_{1}<L_{2}$ and $L_{3}<L_{4}$, then for each $\lambda \in\left(L_{1}, L_{2}\right)$ and $\mu \in\left(L_{3}, L_{4}\right)$ there exists a positive solution $(u(t), v(t)), t \in[0,1]$ for (S)-(BC).

2) If $f_{0}^{s}=0, g_{0}^{S}, f_{\infty}^{i}, g_{\infty}^{i} \in(0, \infty)$, and $L_{3}<L_{4}^{\prime}$, then for each $\lambda \in\left(L_{1}, \infty\right)$ and $\mu \in\left(L_{3}, L_{4}^{\prime}\right)$ there exists a positive solution $(u(t), v(t)), t \in[0,1]$ for (S)-(BC).

3) If $g_{0}^{s}=0, f_{0}^{s}, f_{\infty}^{i}, g_{\infty}^{i} \in(0, \infty)$, and $L_{1}<L_{2}^{\prime}$, then for each $\lambda \in\left(L_{1}, L_{2}^{\prime}\right)$ and $\mu \in\left(L_{3}, \infty\right)$ there exists a positive solution $(u(t), v(t)), t \in[0,1]$ for (S)-(BC).

4) If $f_{0}^{s}=g_{0}^{s}=0, f_{\infty}^{i}, g_{\infty}^{i} \in(0, \infty)$, then for each $\lambda \in\left(L_{1}, \infty\right)$ and $\mu \in\left(L_{3}, \infty\right)$ there exists a positive solution $(u(t), v(t)), t \in[0,1]$ for $(\mathrm{S})-(\mathrm{BC})$.

5) If $f_{0}^{S}, g_{0}^{S} \in(0, \infty)$, and at least one of $f_{\infty}^{i}, g_{\infty}^{i}$ is $\infty$, then for each $\lambda \in\left(0, L_{2}\right)$ and $\mu \in\left(0, L_{4}\right)$ there exists a positive solution $(u(t), v(t)), t \in[0,1]$ for (S)-(BC).

6) If $f_{0}^{s}=0, g_{0}^{s} \in(0, \infty)$, and at least one of $f_{\infty}^{i}, g_{\infty}^{i}$ is $\infty$, then for each $\lambda \in(0, \infty)$ and $\mu \in\left(0, L_{4}^{\prime}\right)$ there exists a positive solution $(u(t), v(t)), t \in[0,1]$ for (S)-(BC).

7) If $f_{0}^{s} \in(0, \infty), g_{0}^{s}=0$, and at least one of $f_{\infty}^{i}, g_{\infty}^{i}$ is $\infty$, then for each $\lambda \in\left(0, L_{2}^{\prime}\right)$ and $\mu \in(0, \infty)$ there exists a positive solution $(u(t), v(t)), t \in[0,1]$ for (S)-(BC).

8) If $f_{0}^{s}=g_{0}^{s}=0$, and at least one of $f_{\infty}^{i}, g_{\infty}^{i}$ is $\infty$, then for each $\lambda \in(0, \infty)$ and $\mu \in(0, \infty)$ there exists a positive solution $(u(t), v(t)), t \in[0,1]$ for (S)-(BC).

Proof. We consider the above cone $P \subset Y$ and the operators $Q_{1}, Q_{2}$ and $Q$. Because the proofs of the above cases are similar, in what follows we will prove one of them, namely Case 3). So, we suppose $g_{0}^{s}=0$ and $f_{0}^{s}, f_{\infty}^{i}, g_{\infty}^{i} \in(0, \infty)$. Let $\lambda \in\left(L_{1}, L_{2}^{\prime}\right)$ and $\mu \in\left(L_{3}, \infty\right)$. We choose $\tilde{\alpha}_{3} \in\left(0,1-\frac{\lambda f_{0}^{s} A}{b}\right)$ and $\tilde{\alpha}_{4} \in\left(0,1-\frac{\lambda f_{0}^{s} D}{1-b}\right)$. Let $\varepsilon>0$ be a positive number such that $\varepsilon<f_{\infty}^{i}, \varepsilon<g_{\infty}^{i}$ and

$$
\begin{gathered}
\frac{a \alpha_{1}}{\gamma \gamma_{1}\left(f_{\infty}^{i}-\varepsilon\right) \tilde{A}} \leq \lambda, \quad \frac{a\left(1-\alpha_{1}\right)}{\gamma \gamma_{1}\left(g_{\infty}^{i}-\varepsilon\right) \tilde{B}} \leq \mu, \quad \frac{(1-a) \alpha_{2}}{\gamma \gamma_{2}\left(f_{\infty}^{i}-\varepsilon\right) \widetilde{D}} \leq \lambda, \quad \frac{(1-a)\left(1-\alpha_{2}\right)}{\gamma \gamma_{2}\left(g_{\infty}^{i}-\varepsilon\right) \tilde{C}} \leq \mu, \\
\frac{b\left(1-\tilde{\alpha}_{3}\right)}{\left(f_{0}^{s}+\varepsilon\right) A} \geq \lambda, \quad \frac{b \tilde{\alpha}_{3}}{\varepsilon B} \geq \mu, \quad \frac{(1-b)\left(1-\tilde{\alpha}_{4}\right)}{\left(f_{0}^{s}+\varepsilon\right) D} \geq \lambda, \quad \frac{(1-b) \tilde{\alpha}_{4}}{\varepsilon C} \geq \mu .
\end{gathered}
$$


By using (H2) and the definitions of $f_{0}^{s}$ and $g_{0}^{s}$, we deduce that there exists $R_{1}>0$ such that $f(t, u, v) \leq\left(f_{0}^{s}+\varepsilon\right)(u+v)$ and $g(t, u, v) \leq \varepsilon(u+v)$ for all $t \in[0,1], u, v \in[0, \infty)$ with $0 \leq u+v \leq$ $R_{1}$. We define the set $\Omega_{1}=\left\{(u, v) \in Y,\|(u, v)\|_{Y}<R_{1}\right\}$. Now let $(u, v) \in P \cap \partial \Omega_{1}$, that is $(u, v) \in P$ with $\|(u, v)\|_{Y}=R_{1}$ or equivalently $\|u\|+\|v\|=R_{1}$. Then $u(t)+v(t) \leq R_{1}$ for all $t \in[0,1]$, and by Lemma 2.3, we obtain

$$
\begin{gathered}
Q_{1}(u, v)(t) \leq \lambda \int_{0}^{1} J_{1}(s) f(s, u(s), v(s)) d s+\mu \int_{0}^{1} J_{2}(s) g(s, u(s), v(s)) d s \\
\leq \lambda \int_{0}^{1} J_{1}(s)\left(f_{0}^{s}+\varepsilon\right)(u(s)+v(s)) d s+\mu \int_{0}^{1} J_{2}(s) \varepsilon(u(s)+v(s)) d s \\
\leq \lambda\left(f_{0}^{s}+\varepsilon\right) \int_{0}^{1} J_{1}(s)(\|u\|+\|v\|) d s+\mu \varepsilon \int_{0}^{1} J_{2}(s)(\|u\|+\|v\|) d s \\
=\left[\lambda\left(f_{0}^{s}+\varepsilon\right) A+\mu \varepsilon B\right]\|(u, v)\|_{Y} \leq\left[b\left(1-\tilde{\alpha}_{3}\right)+b \tilde{\alpha}_{3}\right]\|(u, v)\|_{Y}=b\|(u, v)\|_{Y}, \quad \forall t \in[0,1] .
\end{gathered}
$$

Therefore $\left\|Q_{1}(u, v)\right\| \leq b\|(u, v)\|_{Y}$.

In a similar manner, we conclude

$$
\begin{gathered}
Q_{2}(u, v)(t) \leq \mu \int_{0}^{1} J_{3}(s) g(s, u(s), v(s)) d s+\lambda \int_{0}^{1} J_{4}(s) f(s, u(s), v(s)) d s \\
\leq \mu \int_{0}^{1} J_{3}(s) \varepsilon(u(s)+v(s)) d s+\lambda \int_{0}^{1} J_{4}(s)\left(f_{0}^{s}+\varepsilon\right)(u(s)+v(s)) d s \\
\leq \mu \varepsilon \int_{0}^{1} J_{3}(s)(\|u\|+\|v\|) d s+\lambda\left(f_{0}^{s}+\varepsilon\right) \int_{0}^{1} J_{4}(s)(\|u\|+\|v\|) d s \\
=\left[\mu \varepsilon C+\lambda\left(f_{0}^{s}+\varepsilon\right) D\right]\|(u, v)\|_{Y} \leq\left[(1-b) \tilde{\alpha}_{4}+(1-b)\left(1-\tilde{\alpha}_{4}\right)\right]\|(u, v)\|_{Y} \\
=(1-b)\|(u, v)\|_{Y}, \quad \forall t \in[0,1] .
\end{gathered}
$$

Hence $\left\|Q_{2}(u, v)\right\| \leq(1-b)\|(u, v)\|_{Y}$.

Then, for $(u, v) \in P \cap \partial \Omega_{1}$, we deduce

$$
\|Q(u, v)\|_{Y}=\left\|Q_{1}(u, v)\right\|+\left\|Q_{2}(u, v)\right\| \leq b\|(u, v)\|_{Y}+(1-b)\|(u, v)\|_{Y}=\|(u, v)\|_{Y} .
$$

By the definitions of $f_{\infty}^{i}$ and $g_{\infty}^{i}$, there exists $\bar{R}_{2}>0$ such that $f(t, u, v) \geq\left(f_{\infty}^{i}-\varepsilon\right)(u+v)$ and $g(t, u, v) \geq\left(g_{\infty}^{i}-\varepsilon\right)(u+v)$ for all $u, v \geq 0$ with $u+v \geq \bar{R}_{2}$ and $t \in\left[c_{1}, c_{2}\right]$. We consider $R_{2}=$ $\max \left\{2 R_{1}, \bar{R}_{2} / \gamma\right\}$ and we define $\Omega_{2}=\left\{(u, v) \in Y,\|(u, v)\|_{Y}<R_{2}\right\}$. Then for $(u, v) \in P$ with $\|(u, v)\|_{Y}=$ $R_{2}$, we obtain $u(t)+v(t) \geq t^{\alpha-1}\|u\|+t^{\beta-1}\|v\| \geq \gamma\|(u, v)\|_{Y}=\gamma R_{2} \geq \bar{R}_{2}$, for all $t \in\left[c_{1}, c_{2}\right]$.

Then by Lemma 2.3, we conclude

$$
\begin{gathered}
Q_{1}(u, v)\left(c_{1}\right) \geq \lambda c_{1}^{\alpha-1} \int_{c_{1}}^{c_{2}} J_{1}(s) f(s, u(s), v(s)) d s+\mu c_{1}^{\alpha-1} \int_{c_{1}}^{c_{2}} J_{2}(s) g(s, u(s), v(s)) d s \\
\geq \lambda \gamma_{1} \int_{c_{1}}^{c_{2}} J_{1}(s)\left(f_{\infty}^{i}-\varepsilon\right)(u(s)+v(s)) d s+\mu \gamma_{1} \int_{c_{1}}^{c_{2}} J_{2}(s)\left(g_{\infty}^{i}-\varepsilon\right)(u(s)+v(s)) d s \\
\geq \lambda \gamma \gamma_{1}\left(f_{\infty}^{i}-\varepsilon\right) \int_{c_{1}}^{c_{2}} J_{1}(s)\|(u, v)\|_{Y} d s+\mu \gamma \gamma_{1}\left(g_{\infty}^{i}-\varepsilon\right) \int_{c_{1}}^{c_{2}} J_{2}(s)\|(u, v)\|_{Y} d s \\
=\left[\lambda \gamma \gamma_{1}\left(f_{\infty}^{i}-\varepsilon\right) \tilde{A}+\mu \gamma \gamma_{1}\left(g_{\infty}^{i}-\varepsilon\right) \tilde{B}\right]\|(u, v)\|_{Y}
\end{gathered}
$$




$$
\geq\left[a \alpha_{1}+a\left(1-\alpha_{1}\right)\right]\|(u, v)\|_{Y}=a\|(u, v)\|_{Y}
$$

So, $\left\|Q_{1}(u, v)\right\| \geq Q_{1}(u, v)\left(c_{1}\right) \geq a\|(u, v)\|_{Y}$.

In a similar manner, we conclude

$$
\begin{gathered}
Q_{2}(u, v)\left(c_{1}\right) \geq \mu c_{1}^{\beta-1} \int_{c_{1}}^{c_{2}} J_{3}(s) g(s, u(s), v(s)) d s+\lambda c_{1}^{\beta-1} \int_{c_{1}}^{c_{2}} J_{4}(s) f(s, u(s), v(s)) d s \\
\geq \mu \gamma_{2} \int_{c_{1}}^{c_{2}} J_{3}(s)\left(g_{\infty}^{i}-\varepsilon\right)(u(s)+v(s)) d s+\lambda \gamma_{2} \int_{c_{1}}^{c_{2}} J_{4}(s)\left(f_{\infty}^{i}-\varepsilon\right)(u(s)+v(s)) d s \\
\geq \mu \gamma \gamma_{2}\left(g_{\infty}^{i}-\varepsilon\right) \int_{c_{1}}^{c_{2}} J_{3}(s)\|(u, v)\|_{Y} d s+\lambda \gamma \gamma_{2}\left(f_{\infty}^{i}-\varepsilon\right) \int_{c_{1}}^{c_{2}} J_{4}(s)\|(u, v)\|_{Y} d s \\
=\left[\mu \gamma \gamma_{2}\left(g_{\infty}^{i}-\varepsilon\right) \tilde{C}+\lambda \gamma \gamma_{2}\left(f_{\infty}^{i}-\varepsilon\right) \widetilde{D}\right]\|(u, v)\|_{Y} \\
\geq\left[(1-a)\left(1-\alpha_{2}\right)+(1-a) \alpha_{2}\right]\|(u, v)\|_{Y}=(1-a)\|(u, v)\|_{Y} .
\end{gathered}
$$

So, $\left\|Q_{2}(u, v)\right\| \geq Q_{2}(u, v)\left(c_{1}\right) \geq(1-a)\|(u, v)\|_{Y}$.

Hence, for $(u, v) \in P \cap \partial \Omega_{2}$, we obtain

$$
\|Q(u, v)\|_{Y}=\left\|Q_{1}(u, v)\right\|+\left\|Q_{2}(u, v)\right\| \geq a\|(u, v)\|_{Y}+(1-a)\|(u, v)\|_{Y}=\|(u, v)\|_{Y}
$$

By using (6), (7) and Theorem 2.1, i), we conclude that $Q$ has a fixed point $(u, v) \in P \cap\left(\overline{\Omega_{2}} \backslash \Omega_{1}\right)$ such that $R_{1} \leq\|u\|+\|v\| \leq R_{2}, u(t) \geq t^{\alpha-1}\|u\|, v(t) \geq t^{\beta-1}\|v\|$ for all $t \in[0,1]$. If $\|u\|>0$ then $u(t)>0$ for all $t \in(0,1]$, and if $\|v\|>0$ then $v(t)>0$ for all $t \in(0,1]$.

In what follows, for $f_{0}^{i}, g_{0}^{i}, f_{\infty}^{s}, g_{\infty}^{s} \in(0, \infty)$ and numbers $\alpha_{1}, \alpha_{2} \in[0,1], \alpha_{3}, \alpha_{4} \in(0,1), a \in[0,1]$ and $b \in(0,1)$, we define the numbers

$$
\begin{gathered}
\tilde{L}_{1}=\max \left\{\frac{a \alpha_{1}}{\gamma \gamma_{1} f_{0}^{i} \tilde{A}}, \frac{(1-a) \alpha_{2}}{\gamma \gamma_{2} f_{0}^{i} \widetilde{D}}\right\}, \quad \tilde{L}_{2}=\min \left\{\frac{b \alpha_{3}}{f_{\infty}^{s} A}, \frac{(1-b) \alpha_{4}}{f_{\infty}^{s} D}\right\}, \\
\tilde{L}_{3}=\max \left\{\frac{a\left(1-\alpha_{1}\right)}{\gamma \gamma_{1} g_{0}^{i} \tilde{B}}, \frac{(1-a)\left(1-\alpha_{2}\right)}{\gamma \gamma_{2} g_{0}^{i} \tilde{C}}\right\}, \quad \tilde{L}_{4}=\min \left\{\frac{b\left(1-\alpha_{3}\right)}{g_{\infty}^{s} B}, \frac{(1-b)\left(1-\alpha_{4}\right)}{g_{\infty}^{s} C}\right\}, \\
\tilde{L}_{2}^{\prime}=\min \left\{\frac{b}{f_{\infty}^{s} A}, \frac{1-b}{f_{\infty}^{s} D}\right\}, \quad \tilde{L}_{4}^{\prime}=\min \left\{\frac{b}{g_{\infty}^{s} B}, \frac{1-b}{g_{\infty}^{S} C}\right\} .
\end{gathered}
$$

By using similar arguments as those used in the proof of Theorem 3.1 (see also [5]) we obtain the following result.

Theorem 3.2 Assume that (H1) and (H2) hold, $\left[c_{1}, c_{2}\right] \subset[0,1]$ with $0<c_{1}<c_{2} \leq 1, \alpha_{1}, \alpha_{2} \in[0,1]$, $\alpha_{3}, \alpha_{4} \in(0,1), a \in[0,1]$ and $b \in(0,1)$.

1) If $f_{0}^{i}, g_{0}^{i}, f_{\infty}^{s}, g_{\infty}^{s} \in(0, \infty), \tilde{L}_{1}<\tilde{L}_{2}$ and $\tilde{L}_{3}<\tilde{L}_{4}$ then for each $\lambda \in\left(\tilde{L}_{1}, \tilde{L}_{2}\right)$ and $\mu \in\left(\tilde{L}_{3}, \tilde{L}_{4}\right)$ there exists a positive solution $(u(t), v(t)), t \in[0,1]$ for (S)-(BC).

2) If $f_{0}^{i}, g_{0}^{i}, f_{\infty}^{s} \in(0, \infty), g_{\infty}^{s}=0$ and $\tilde{L}_{1}<\tilde{L}_{2}^{\prime}$, then for each $\lambda \in\left(\tilde{L}_{1}, \tilde{L}_{2}^{\prime}\right)$ and $\mu \in\left(\tilde{L}_{3}, \infty\right)$ there exists a positive solution $(u(t), v(t)), t \in[0,1]$ for (S)-(BC).

3) If $f_{0}^{i}, g_{0}^{i}, g_{\infty}^{s} \in(0, \infty), f_{\infty}^{s}=0$, and $\tilde{L}_{3}<\tilde{L}_{4}^{\prime}$, then for each $\lambda \in\left(\tilde{L}_{1}, \infty\right)$ and $\mu \in\left(\tilde{L}_{3}, \tilde{L}_{4}^{\prime}\right)$ there exists a positive solution $(u(t), v(t)), t \in[0,1]$ for (S)-(BC).

4) If $f_{0}^{i}, g_{0}^{i} \in(0, \infty), f_{\infty}^{s}=g_{\infty}^{S}=0$, then for each $\lambda \in\left(\tilde{L}_{1}, \infty\right)$ and $\mu \in\left(\tilde{L}_{3}, \infty\right)$ there exists a positive solution $(u(t), v(t)), t \in[0,1]$ for (S)-(BC). 
5) If $f_{\infty}^{s}, g_{\infty}^{s} \in(0, \infty)$, and at least one of $f_{0}^{i}, g_{0}^{i}$ is $\infty$, then for each $\lambda \in\left(0, \tilde{L}_{2}\right)$ and $\mu \in\left(0, \tilde{L}_{4}\right)$ there exists a positive solution $(u(t), v(t)), t \in[0,1]$ for (S)-(BC).

6) If $f_{\infty}^{s} \in(0, \infty), g_{\infty}^{s}=0$ and at least one of $f_{0}^{i}, g_{0}^{i}$ is $\infty$, then for each $\lambda \in\left(0, \tilde{L}_{2}^{\prime}\right)$ and $\mu \in(0, \infty)$ there exists a positive solution $(u(t), v(t)), t \in[0,1]$ for (S)-(BC).

7) If $f_{\infty}^{s}=0, g_{\infty}^{s} \in(0, \infty)$ and at least one of $f_{0}^{i}, g_{0}^{i}$ is $\infty$, then for each $\lambda \in(0, \infty)$ and $\mu \in\left(0, \tilde{L}_{4}^{\prime}\right)$ there exists a positive solution $(u(t), v(t)), t \in[0,1]$ for (S)-(BC).

8) If $f_{\infty}^{s}=g_{\infty}^{s}=0$ and at least one of $f_{0}^{i}, g_{0}^{i}$ is $\infty$, then for each $\lambda \in(0, \infty)$ and $\mu \in(0, \infty)$ there exists a positive solution $(u(t), v(t)), t \in[0,1]$ for (S)-(BC).

Next we present intervals for $\lambda$ and $\mu$ for which there exists no positive solutions of problem (S)-(BC), viewed as fixed points of operator $Q$.

Theorem 3.3 Assume that (H1) and (H2) hold. If there exist positive numbers $M_{1}, M_{2}$ such that

$$
f(t, u, v) \leq M_{1}(u+v), g(t, u, v) \leq M_{2}(u+v), \forall t \in[0,1], u, v \geq 0,
$$

then there exist positive constants $\lambda_{0}$ and $\mu_{0}$ such that for every $\lambda \in\left(0, \lambda_{0}\right), \mu \in\left(0, \mu_{0}\right)$ the boundary value problem $(\mathrm{S})-(\mathrm{BC})$ has no positive solution.

In the proof of Theorem 3.3 we can show that $\lambda_{0}=\min \left\{\frac{1}{4 M_{1} A}, \frac{1}{4 M_{1} D}\right\}$ and $\mu_{0}=\min \left\{\frac{1}{4 M_{2} B}, \frac{1}{4 M_{2} C}\right\}$, where $A=\int_{0}^{1} J_{1}(s) d s, B=\int_{0}^{1} J_{2}(s) d s, C=\int_{0}^{1} J_{3}(s) d s, D=\int_{0}^{1} J_{4}(s) d s$, satisfy the conditions of our theorem. If $f_{0}^{s}, g_{0}^{s}, f_{\infty}^{s}, g_{\infty}^{s}<\infty$, then there exist positive constants $M_{1}$ and $M_{2}$ such that (8) holds, and then we obtain the conclusion of Theorem 3.3.

Theorem 3.4 a) Assume that (H1) and (H2) hold. If there exist positive numbers $c_{1}, c_{2}$ with $0<c_{1}<$ $c_{2} \leq 1$ and $m_{1}>0$ such that

$$
f(t, u, v) \geq m_{1}(u+v), \forall t \in\left[c_{1}, c_{2}\right], u, v \geq 0,
$$

then there exists a positive constant $\tilde{\lambda}_{0}$ such that for every $\lambda>\tilde{\lambda}_{0}$ and $\mu>0$, the boundary value problem (S)-(BC) has no positive solution.

b) Assume that (H1) and (H2) hold. If there exist positive numbers $c_{1}, c_{2}$ with $0<c_{1}<c_{2} \leq 1$ and $m_{2}>0$ such that

$$
g(t, u, v) \geq m_{2}(u+v), \forall t \in\left[c_{1}, c_{2}\right], u, v \geq 0,
$$

then there exists a positive constant $\tilde{\mu}_{0}$ such that for every $\lambda>0$ and $\mu>\tilde{\mu}_{0}$, the boundary value problem (S)-(BC) has no positive solution.

c) Assume that (H1) and (H2) hold. If there exist positive numbers $c_{1}, c_{2}$ with $0<c_{1}<c_{2} \leq 1$ and $m_{1}, m_{2}>0$ such that

$$
f(t, u, v) \geq m_{1}(u+v), g(t, u, v) \geq m_{2}(u+v), \forall t \in\left[c_{1}, c_{2}\right], u, v \geq 0,
$$

then there exists positive constants $\hat{\lambda}_{0}$ and $\hat{\mu}_{0}$ such that for every $\lambda>\hat{\lambda}_{0}$ and $\mu>\hat{\mu}_{0}$, the boundary value problem (S)-(BC) has no positive solution.

In the proof of Theorem 3.4 we define $\tilde{\lambda}_{0}=\min \left\{\frac{1}{\gamma \gamma_{1} m_{1} \tilde{A}}, \frac{1}{\gamma \gamma_{2} m_{1} \tilde{D}}\right\}, \tilde{\mu}_{0}=\min \left\{\frac{1}{\gamma \gamma_{1} m_{2} \tilde{B}}, \frac{1}{\gamma \gamma_{2} m_{2} \tilde{C}}\right\}$, where $\tilde{A}=\int_{c_{1}}^{c_{2}} J_{1}(s) d s, \tilde{B}=\int_{c_{1}}^{c_{2}} J_{2}(s) d s, \tilde{C}=\int_{c_{1}}^{c_{2}} J_{3}(s) d s, \widetilde{D}=\int_{c_{1}}^{c_{2}} J_{4}(s) d s$, and $\hat{\lambda}_{0}=\frac{1}{2 \gamma \gamma_{1} m_{1} \tilde{A}^{\prime}}, \hat{\mu}_{0}=\frac{1}{2 \gamma \gamma_{2} m_{2} \tilde{C}^{*}}$

If for $c_{1}, c_{2}$ with $0<c_{1}<c_{2} \leq 1, f_{0}^{i}, f_{\infty}^{i}>0$ and $f(t, u, v)>0$ for all $t \in\left[c_{1}, c_{2}\right]$ and $u, v \geq 0$ with 
$u+v>0$, then relation (9) holds and we obtain the conclusion of Theorem 3.4 a). If for $c_{1}, c_{2}$ with $0<\mathrm{c}_{1}<\mathrm{c}_{2} \leq 1, g_{0}^{i}, g_{\infty}^{i}>0$ and $g(t, u, v)>0$ for all $t \in\left[c_{1}, c_{2}\right]$ and $u, v \geq 0$ with $u+v>0$, then relation (10) holds and we obtain the conclusion of Theorem $3.4 \mathrm{~b}$ ). If for $c_{1}, c_{2}$ with $0<\mathrm{c}_{1}<\mathrm{c}_{2} \leq 1$, $f_{0}^{i}, f_{\infty}^{i}, g_{0}^{i}, g_{\infty}^{i}>0$ and $f(t, u, v)>0, g(t, u, v)>0$ for all $t \in\left[c_{1}, c_{2}\right]$ and $u, v \geq 0$ with $u+v>0$, then relation (11) holds and we obtain the conclusion of Theorem $3.4 \mathrm{c}$ ).

\section{An Example}

Let $=\frac{9}{2}(n=5), \quad \beta=\frac{8}{3}(m=3), \quad p_{1}=4 / 3, \quad p_{2}=1, \quad q_{1}=1 / 2, \quad q_{2}=2 / 3, \quad N=1, M=2, \quad \xi_{1}=1 / 2$, $a_{1}=2, \eta_{1}=1 / 3, \eta_{2}=2 / 3, b_{1}=1$ and $b_{2}=1 / 2$.

We consider the system of fractional differential equations

$$
\left\{\begin{array}{c}
D_{0+}^{9 / 2} u(t)+\lambda(t+1)^{\tilde{a}}\left(u^{2}(t)+v^{2}(t)\right)=0, \quad t \in(0,1) \\
D_{0+}^{8 / 3} v(t)+\mu t^{\tilde{b}}\left(e^{u(t)+v(t)}-1\right)=0, \quad t \in(0,1)
\end{array}\right.
$$

with the coupled multi-point boundary conditions

$$
\left(B C_{0}\right) \quad\left\{\begin{array}{c}
u(0)=u^{\prime}(0)=u^{\prime \prime}(0)=u^{\prime \prime \prime}(0)=0,\left.D_{0+}^{4 / 3} u(t)\right|_{t=1}=\left.2 D_{0+}^{1 / 2} v(t)\right|_{t=1 / 2}, \\
v(0)=v^{\prime}(0)=0, v^{\prime}(1)=\left.D_{0+}^{2 / 3} u(t)\right|_{t=1 / 3}+\left.\frac{1}{2} D_{0+}^{2 / 3} u(t)\right|_{t=2 / 3} .
\end{array}\right.
$$

Here we have $f(t, u, v)=(t+1)^{\tilde{a}}\left(u^{2}+v^{2}\right), g(t, u, v)=t^{\tilde{b}}\left(e^{u+v}-1\right)$ for $t \in[0,1], u, v \geq 0$, where $\tilde{a}, \tilde{b}>0$. Then we obtain $\Delta=\frac{5 \Gamma(9 / 2)}{3 \Gamma(19 / 6)}-\frac{\Gamma(9 / 2) \Gamma(8 / 3)\left(1+2^{11 / 6}\right)}{2^{1 / 6} 3^{17 / 6} \Gamma(13 / 6) \Gamma(23 / 6)} \approx 7.6683666$, and so the assumptions (H1) and (H2) are satisfied. In addition, we deduce (see [1])

$$
\begin{aligned}
& g_{1}(t, s)=\frac{1}{\Gamma(9 / 2)}\left\{\begin{array}{c}
t^{7 / 2}(1-s)^{13 / 6}-(t-s)^{7 / 2}, 0 \leq s \leq t \leq 1 \\
t^{7 / 2}(1-s)^{13 / 6}, 0 \leq t \leq s \leq 1
\end{array}\right. \\
& g_{2}(t, s)=\frac{1}{\Gamma(23 / 6)}\left\{\begin{array}{c}
t^{17 / 6}(1-s)^{13 / 6}-(t-s)^{17 / 6}, 0 \leq s \leq t \leq 1 \\
t^{17 / 6}(1-s)^{13 / 6}, 0 \leq t \leq s \leq 1
\end{array}\right. \\
& g_{3}(t, s)=\frac{1}{\Gamma(13 / 6)}\left\{\begin{array}{c}
t^{7 / 6}(1-s)^{2 / 3}-(t-s)^{7 / 6}, 0 \leq s \leq t \leq 1 \\
t^{7 / 6}(1-s)^{2 / 3}, 0 \leq t \leq s \leq 1
\end{array}\right. \\
& g_{4}(t, s)=\frac{1}{\Gamma(8 / 3)}\left\{\begin{array}{c}
t^{5 / 3}(1-s)^{2 / 3}-(t-s)^{5 / 3}, 0 \leq s \leq t \leq 1 \\
t^{5 / 3}(1-s)^{2 / 3}, 0 \leq t \leq s \leq 1
\end{array}\right.
\end{aligned}
$$

$$
h_{1}(s)=\frac{1}{\Gamma(9 / 2)}(1-s)^{13 / 6}\left(1-(1-s)^{4 / 3}\right), h_{2}(s)=\frac{1}{\Gamma(23 / 6)}(1-s)^{13 / 6}\left(1-(1-s)^{2 / 3}\right), h_{3}(s)=
$$
$\frac{1}{\Gamma(13 / 6)}(1-s)^{2 / 3}\left(1-(1-s)^{1 / 2}\right), h_{4}(s)=\frac{1}{\Gamma(8 / 3)} s(1-s)^{2 / 3}$ for all $s \in[0,1]$. For the functions $J_{i}, i=$ $1, \ldots, 4$, we obtain

$$
J_{1}(s)=\left\{\begin{array}{c}
\frac{1}{\Gamma(9 / 2)}(1-s)^{13 / 6}\left(1-(1-s)^{4 / 3}\right)+\frac{\Gamma(8 / 3)}{2^{7 / 6} 3^{17 / 6} \Delta \Gamma(13 / 6) \Gamma(23 / 6)}\left[2(1-s)^{13 / 6}\right. \\
\left.-2(1-3 s)^{17 / 6}+2^{17 / 6}(1-s)^{13 / 6}-(2-3 s)^{17 / 6}\right], \quad 0 \leq s<1 / 3, \\
\frac{1}{\Gamma(9 / 2)}(1-s)^{13 / 6}\left(1-(1-s)^{4 / 3}\right)+\frac{\Gamma(8 / 3)}{2^{7 / 6} 3^{17 / 6} \Delta \Gamma(13 / 6) \Gamma(23 / 6)}\left[2(1-s)^{13 / 6}\right. \\
\left.+2^{17 / 6}(1-s)^{13 / 6}-(2-3 s)^{17 / 6}\right], 1 / 3 \leq s<2 / 3, \\
\frac{1}{\Gamma(9 / 2)}(1-s)^{13 / 6}\left(1-(1-s)^{4 / 3}\right)+\frac{\Gamma(8 / 3)}{2^{1 / 6} 3^{17 / 6} \Delta \Gamma(13 / 6) \Gamma(23 / 6)}\left[(1-s)^{13 / 6}\right. \\
\left.+2^{11 / 6}(1-s)^{13 / 6}\right], \frac{2}{3} \leq s \leq 1,
\end{array}\right.
$$




$$
\begin{aligned}
& J_{2}(s)=\frac{5}{3 \cdot 2^{1 / 6} \Delta \Gamma(13 / 6)}\left\{\begin{array}{c}
(1-s)^{2 / 3}-(1-2 s)^{7 / 6}, \quad 0 \leq s<1 / 2 \\
(1-s)^{2 / 3}, \frac{1}{2} \leq s \leq 1
\end{array}\right. \\
& J_{3}(s)=\left\{\begin{array}{c}
\frac{1}{\Gamma(8 / 3)} s(1-s)^{2 / 3}+\frac{\left(1+2^{11 / 6}\right) \Gamma(9 / 2)}{2^{1 / 6} 3^{17 / 6} \Delta \Gamma(13 / 6) \Gamma(23 / 6)}\left[(1-s)^{2 / 3}\right. \\
\left.-(1-2 s)^{7 / 6}\right], 0 \leq s<1 / 2, \\
\frac{1}{\Gamma(8 / 3)} s(1-s)^{2 / 3}+\frac{\left(1+2^{11 / 6}\right) \Gamma(9 / 2)}{2^{1 / 6} 3^{17 / 6} \Delta \Gamma(13 / 6) \Gamma(23 / 6)}(1-s)^{2 / 3}, \quad 1 / 2 \leq s \leq 1,
\end{array}\right. \\
& J_{4}(s)=\frac{\Gamma(9 / 2)}{2 \cdot 3^{17 / 6} \Delta \Gamma(19 / 6) \Gamma(23 / 6)}\left\{\begin{array}{c}
2(1-s)^{13 / 6}-2(1-3 s)^{17 / 6}+2^{17 / 6}(1-s)^{13 / 6} \\
-(2-3 s)^{17 / 6}, \quad 0 \leq s<1 / 3 \\
2(1-s)^{13 / 6}+2^{17 / 6}(1-s)^{13 / 6} \\
-(2-3 s)^{17 / 6}, \quad 1 / 3 \leq s<2 / 3 \\
2(1-s)^{13 / 6}+2^{17 / 6}(1-s)^{13 / 6}, \quad 2 / 3 \leq s \leq 1 .
\end{array}\right.
\end{aligned}
$$

For $c_{1}=1 / 2, c_{2}=1$, we deduce $\gamma_{1}=(1 / 2)^{7 / 2}, \gamma_{2}=(1 / 2)^{5 / 3}, \gamma=\gamma_{1}$. In addition, we obtain $f_{0}^{s}=0$, $g_{0}^{s}=1, f_{\infty}^{i}=g_{\infty}^{i}=\infty, \quad B=\int_{0}^{1} J_{2}(s) d s \approx 0.06605546, C=\int_{0}^{1} J_{3}(s) d s \approx 0.16869513, \quad \tilde{B}=\int_{1 / 2}^{1} J_{2}(s) d s \approx$ $0.03381002, \tilde{C}=\int_{1 / 2}^{1} J_{3}(s) d s \approx 0.09615861$. Besides, for $b=1 / 2$, we get $L_{4}^{\prime}=\frac{1}{2 C} \approx 2.96392$. Then, by Theorem 3.1,6) we conclude that for each $\lambda \in(0, \infty)$ and $\mu \in\left(0, L_{4}^{\prime}\right)$, there exists a positive solution $(u(t), v(t)), t \in[0,1]$ for problem $\left(S_{0}\right)-\left(B C_{0}\right)$. Because $g_{0}^{i}=2^{-\tilde{b}}$ and $g_{\infty}^{i}=\infty$, we can also apply Theorem $3.4, \mathrm{~b})$. Then there exists $\tilde{\mu}_{0}$ such that for every $\mu>\tilde{\mu}_{0}$ and $\lambda>0$, problem $\left(S_{0}\right)-\left(B C_{0}\right)$ has no positive solution. For example, if $\tilde{b}=1$, then we deduce $m_{2}=\frac{1}{2}$ and $\tilde{\mu}_{0}=\frac{1}{\gamma \gamma_{2} m_{2} \tilde{C}} \approx 747.074$.

\section{References}

[1] Henderson, J., \& Luca, R. (2017). Systems of Riemann-Liouville fractional equations with multi-point boundary conditions, Appl. Math. Comput., 309, 303-323.

[2] Luca, R. (2017). Existence of positive solutions for a fractional boundary value problem. Bul. Inst. Politeh. Iasi, 63(67), (4), 37-49.

[3] Henderson, J., \& Luca, R. (2017). Positive solutions for a system of fractional boundary value problems. Proceedings of EQUADIFF 2017 Conference (pp. 1-10).

[4] Henderson, J., \& Luca, R. (2014). Existence and multiplicity of positive solutions for a system of fractional boundary value problems. Bound. Value Probl., 2014(60), 1-17.

[5] Henderson, J., \& Luca, R. (2014). Positive solutions for a system of fractional differential equations with coupled integral boundary conditions. Appl. Math. Comput., 249, 182-197.

[6] Luca, R., \& Tudorache, A. (2014). Positive solutions to a system of semipositone fractional boundary value problems. Adv. Difference Equ., 2014(179), 1-11.

[7] Henderson, J., \& Luca, R. (2015). Nonexistence of positive solutions for a system of coupled fractional boundary value problems. Bound. Value Probl., 2015(138), 1-12.

[8] Henderson, J., Luca, R., \& Tudorache, A. (2015). On a system of fractional differential equations with coupled integral boundary conditions. Fract. Calc. Appl. Anal., 18(2), 361-386.

[9] Henderson, J., \& Luca, R. (2016). Boundary Value Problems for Systems of Differential, Difference and Fractional Equations. Positive Solutions. Elsevier, Amsterdam. 
[10] Henderson, J., \& Luca, R. (2016). Positive solutions for a system of semipositone coupled fractional Boundary value problems. Bound. Value Probl., 2016(61), 1-23.

[11] Henderson, J., \& Luca, R. (2017). Existence of positive solutions for a singular fractional boundary value problem. Nonlinear Anal. Model. Control, 22(1), 99-114.

[12] Henderson, J., Luca, R., \& Tudorache, A. (2017). Positive solutions for a system of fractional differential equations with multi-point boundary conditions.

[13] Arafa, A. A. M., Rida, S. Z., \& Khalil, M. (2012). Fractional modeling dynamics of HIV and CD4+ T-cells during primary infection. Nonlinear Biomed. Phys., 6(1), 1-7.

[14] Baleanu, D., Diethelm, K., Scalas, E., \& Trujillo, J. J. (2012). Fractional calculus models and numerical methods. Series on Complexity, Nonlinearity and Chaos. World Scientific, Boston.

[15] Cole, K. (1993). Electric conductance of biological systems. Proceedings of Cold Spring Harbor Symp. Quant. Biol., (pp. 107-116). Col Springer Harbor Laboratory Press, New York.

[16] Das, S. (2008). Functional Fractional Calculus for System Identification and Controls. Springer, New York.

[17] Ding, Y., \& Ye, H. (2009). A fractional-order differential equation model of HIV infection of CD4+ T-cells. Math. Comp. Model., 50, 386-392.

[18] Djordjevic, V., Jaric, J., Fabry, B., Fredberg, J., \& Stamenovic, D. (2003). Fractional derivatives embody essential features of cell rheological behavior. Ann. Biomed. Eng., 31, 692-699.

[19] Ge, Z. M., \& Ou, C. Y. (2008). Chaos synchronization of fractional order modified duffing systems with parameters excited by a chaotic signal. Chaos Solitons Fractals, 35, 705-717.

[20] Kilbas, A. A., Srivastava, H. M., \& Trujillo, J. J. (2006). Theory and applications of fractional differential equations. North-Holland Mathematics Studies, 204. Elsevier Science B.V., Amsterdam.

[21] Klafter, J., Lim, S. C., \& Metzler, R. (2011). Fractional Dynamics in Physics. Singapore, World Scientific.

[22] Metzler, R., \& Klafter, J. (2000). The random walks guide to anomalous diffusion: a fractional dynamics approach. Phys. Rep., 339, 1-77.

[23] Ostoja-Starzewski, M. (2007). Towards thermoelasticity of fractal media, J. Therm. Stress., 30, 889-896.

[24] Podlubny, I. (1999). Fractional Differential Equations. Academic Press, San Diego.

[25] Povstenko, Y. Z. (2015). Fractional Thermoelasticity. New York, Springer.

[26] Sabatier, J., Agrawal, O. P., Machado, J. A. T. (2007). Advances in Fractional Calculus: Theoretical Developments and Applications in Physics and Engineering. Springer, Dordrecht.

[27] Samko, S. G., Kilbas, A. A., \& Marichev, O. I. (1993). Fractional Integrals and Derivatives. Theory and Applications. Gordon and Breach, Yverdon.

[28] Sokolov, I. M., Klafter, J., \& Blumen, A. (2002). A fractional kinetics. Phys. Today, 55, 48-54.

[29] Guo, D., \& Lakshmikantham, V. (1988). Nonlinear Problems in Abstract Cones. Academic Press, New York.

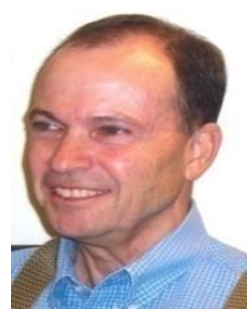

Johnny Henderson is distinguished professor of mathematics at the Baylor University, Waco, Texas, USA. He has also held faculty positions at the Auburn University and the Missouri University of Science and Technology. His published research is primarily in the areas of boundary value problems for ordinary differential equations, finite difference equations, functional differential equations, and dynamic equations on time scales. He is an Inaugural Fellow of the American Mathematical Society. 


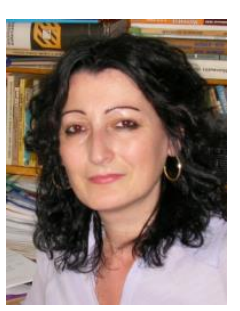

Rodica Luca is professor of mathematics at the "Gheorghe Asachi" Technical University of Iasi, Romania. She obtained her PhD degree in mathematics from "Alexandru Ioan Cuza" University of Iasi. Her research interests are boundary value problems for nonlinear systems of ordinary differential equations, finite difference equations, and fractional differential equations, and initial-boundary value problems for nonlinear hyperbolic systems of partial differential equations.

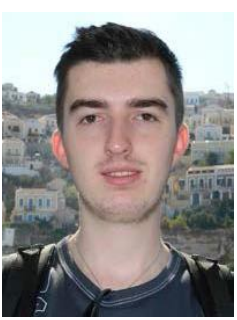

Alexandru Tudorache is graduate student at "Gheorghe Asachi" Technical University of Iasi, Faculty of Computer Engineering and Automatic Control. 